\title{
SOLUTION OF THE BAIRE ORDER PROBLEM OF MAULDIN
}

\author{
MAREK BALCERZAK AND DOROTA ROGOWSKA
}

(Communicated by C. D. Sogge)

\begin{abstract}
Let $X$ be an uncountable Polish space, and let $I$ be a proper $\sigma$-ideal of subsets of $X$ such that $\{x\} \in I$ for each $x \in X$. Denote by $B_{\alpha}(I), \alpha \leq \omega_{1}$, the Baire system generated by the family of functions $f$ : $X \rightarrow \mathbb{R}$ continuous $I$ almost everywhere. We prove that if $r(I)=\min \{\alpha \leq$ $\left.\omega_{1}: B_{\alpha+1}(I)=B_{\alpha}(I)\right\}$, then either $r(I)=1$ or $r(I)=\omega_{1}$. This answers the problem raised by R. D. Mauldin in 1973.
\end{abstract}

\section{INTRODUCTION}

Let $X$ be an uncountable separable and complete metric space (briefly called Polish), and let $I$ be a $\sigma$-ideal of subsets of $X$ such that $X \notin I$. Denote by $C_{I}$ the family of all functions $f: X \rightarrow \mathbb{R}$ whose sets of points of discontinuity are in $I$. Then put $B_{0}(I)=C_{I}$ and for each ordinal $\alpha>0$ define $B_{\alpha}(I)$ as the family of all pointwise limits of sequences of functions from $\bigcup_{\gamma<\alpha} B_{\gamma}(I)$.

It is easy to check that the Baire system $B_{\alpha}(I), \alpha \leq \omega_{1}$, has the following properties:

- $B_{\omega_{1}}(I)$ is closed under pointwise limits, i.e. $B_{\omega_{1}+1}(I)=B_{\omega_{1}}(I)$,

- for $I=\{\emptyset\}$ we have the classical Baire system (denoted by $B_{\alpha}, \alpha \leq \omega_{1}$ ).

Now we define $r(I)=\min \left\{\alpha \leq \omega_{1}: B_{\alpha+1}(I)=B_{\alpha}(I)\right\}$ which is called the Baire order of $C_{I}$.

The following results are known:

(1) If $I=\{\emptyset\}$, then $r(I)=\omega_{1}$ (see [L]).

(2) If $I$ is the $\sigma$-ideal of the first category sets in $X$, then $r(I)=1$ and $B_{1}(I)$ consists of all functions with the Baire property (see [K1]).

(3) If $I$ is the $\sigma$-ideal of sets of Lebesgue measure zero in $[0,1]$, then $r(I)=\omega_{1}$ (see [M2]; for some generalizations compare [M3], [B1]).

In [M2] Mauldin posed the following problem: If $0<\alpha<\omega_{1}$, is there a $\sigma$-ideal $I_{\alpha}$ of the first category subsets of $[0,1]$ which contains all $F_{\sigma}$ sets of Lebesgue measure 0 such that the family of all functions which are continuous except for a set in this $\sigma$-ideal $I_{\alpha}$ has Baire order $\alpha$ ?

Note that in the above question, $\sigma$-ideals are required to contain all singletons $\{x\}$; a $\sigma$-ideal which has that property is called uniform. In the main theorem

Received by the editors April 28, 1994.

1991 Mathematics Subject Classification. Primary 26A21; Secondary 04A15.

Key words and phrases. $\sigma$-ideals, Baire classification, sets of the first category. 
we will show that, for each uniform $\sigma$-ideal $I$ of subsets of $X$, we have either $r(I)=1$ or $r(I)=\omega_{1}$. It solves Mauldin's problem in the negative. Observe that always $r(I)>0$ since the characteristic function of a countable set dense in $X$ belongs to $B_{1}(I) \backslash B_{0}(I)$ (cf. [B2]).

Denote by $\mathscr{B}$ the family of all Borel subsets of $X$, and by $\Sigma_{\alpha}^{0}, \Pi_{\alpha}^{0}$ (for $\left.0<\alpha<\omega_{1}\right)$ the subclasses of $\mathscr{B}$ defined as in [Mo, $\left.1 \mathrm{~F}\right]$. In particular, $\Sigma_{2}^{0}$ is the family of all $F_{\sigma}$ sets in $X$.

A $\sigma$-ideal $I$ is called $\Sigma_{2}^{0}$ supported if each $A \in I$ is contained in some $B \in I \cap \Sigma_{2}^{0}$. For a $\sigma$-ideal $I$, we define

$$
I^{*}=\left\{A \subset X:\left(\exists B \in I \cap \Sigma_{2}^{0}\right)(A \subset B)\right\} .
$$

Obviously, $I^{*}$ is a $\Sigma_{2}^{0}$ supported $\sigma$-ideal, and if $I$ is a $\Sigma_{2}^{0}$ supported $\sigma$-ideal, then $I=I^{*}$. Since the set of discontinuity points of an arbitrary function is of type $F_{\sigma}$, we have $C_{I}=C_{I^{*}}$ for each $\sigma$-ideal $I$, and thus the Baire order problem may be restricted to $\Sigma_{2}^{0}$ supported $\sigma$-ideals.

\section{AUXILIARY FACTS}

In this section we will assume that $I$ is a uniform $\sigma$-ideal of subsets of $X$. If $\mathscr{F}$ is a family of subsets of $X$, then define $\operatorname{MGR}(\mathscr{F})$ as the family of all subsets $B$ of $X$ such that for each $A \in \mathscr{F}$ the set $B \cap A$ is of the first category in $A$.

The following deep result plays a key role in the proof of our Main Theorem.

Proposition 1 [KS, Theorem 2]. Let I be a $\Sigma_{2}^{0}$ supported $\sigma$-ideal. Then precisely one of the following posibilities holds:

(i) $I=M G R(\mathscr{F})$ for a countable family $\mathscr{F}$ of closed subsets of $X$ (moreover, it may be assumed that $\mathscr{F}=\left\{F_{\gamma}: \gamma<\alpha\right\}$ where $\alpha<\omega_{1}$ and $F_{\gamma} \subset F_{\beta}$ for $\beta<\gamma<\alpha$, and $F_{\gamma+1}$ is nowhere dense in $F_{\gamma}$ for $\gamma<\alpha$ );

(ii) there exists a homeomorphic embedding $\varphi: 2^{\omega} \times \omega^{\omega} \rightarrow X$ such that $\varphi\left[\{t\} \times \omega^{\omega}\right] \notin I$ for each $t \in 2^{\omega}$.

Proposition 2. If a $\sigma$-ideal I satisfies condition (ii) of Proposition 1, then I has the following property:

(M) There exists a Borel function $f: X \rightarrow X$ such that $f^{-1}[\{x\}] \notin I$ for each $x \in X$.

Proof. Denote $B=\varphi\left[2^{\omega} \times \omega^{\omega}\right]$ and consider a continuous function $\psi=p r_{1} \circ$ $\varphi^{-1}: B \rightarrow 2^{\omega}$, where $p r_{1}$ is a projection onto the first factor. For each $t \in 2^{\omega}$ we have

$$
\psi^{-1}[\{t\}]=\varphi\left[p r_{1}^{-1}[\{t\}]\right]=\varphi\left[\{t\} \times \omega^{\omega}\right] \notin I .
$$

Since $B$ is a Borel set (even of type $G_{\delta}$, see $[\mathrm{K}, \S 35$, III]), we can extend $\psi$ to a Borel function $g: X \rightarrow 2^{\omega}$ and we get $g^{-1}[\{t\}] \notin I$ for each $t \in 2^{\omega}$. Let $h: 2^{\omega} \rightarrow X$ be a Borel isomorphism (see [K, §37, II]). Then $f=h \circ g: X \rightarrow X$ is a Borel function and for each $x \in X$ we have

$$
f^{-1}[\{x\}]=g^{-1}\left[h^{-1}[\{x\}]\right] \notin I .
$$

Define $R(I)=\min \left\{\alpha \leq \omega_{1}:(\forall B \in \mathscr{B})\left(\exists A \in \Sigma_{\alpha}^{0}\right)(B \triangle A \in I)\right\}$ where $\Sigma_{\omega_{1}}^{0}=\mathscr{B}$ and $B \triangle A=(B \backslash A) \cup(A \backslash B)$. Observe that $\Sigma_{\alpha}^{0}$ can be replaced by $\Pi_{\alpha}^{0}$ in the above definition. 
Proposition 3 [B3, Corollary 2.2]. If a $\sigma$-ideal I has the property (M), then $R(I)=\omega_{1}$.

Proposition 4 [M2, Theorem 3]. For every $\sigma$-ideal $I$ and each $\alpha, 0<\alpha \leq \omega_{1}$, we have $f \in B_{\alpha}(I)$ if and only if there is $g \in B_{\alpha}$ such that $\{x \in X: f(x) \neq$ $g(x)\} \in I^{*}$.

Proposition 5 [B2]. If $I$ is a $\Sigma_{2}^{0}$ supported $\sigma$-ideal and $R(I)=\omega_{1}$, then $r(I)=$ $\omega_{1}$.

Proof. Suppose to the contrary that $r(I)=\alpha<\omega_{1}$, and consider an arbitrary set $E \in \mathscr{B}$. Then Proposition 4 yields that for each Borel function $f: X \rightarrow \mathbb{R}$ there is a function $g \in B_{\alpha}$ satisfying $\{x \in X: f(x) \neq g(x)\} \in I$. In particular, consider such a function $g$ for $f=\chi_{E}$. Put $A=g^{-1}[\{1\}]$. Then $A \in \Pi_{\alpha+1}^{0}$ (cf. [K, $\S 31, \mathrm{IX}]$ ) and $E \triangle A \subset\{x \in X: f(x) \neq g(x)\} \in I$. Hence $R(I) \leq$ $\alpha+1<\omega_{1}$, which gives a contradiction.

\section{MAIN TheOREM}

Theorem. If $X$ is an uncountable Polish space and $I$ is an uniform $\sigma$-ideal, then either $r(I)=1$ or $r(I)=\omega_{1}$.

Proof. According to our general remarks in Introduction, we may assume that $I$ is $\Sigma_{2}^{0}$ supported and, by Proposition 1, we consider Cases (i) and (ii).

Case (i). Assume that $\mathscr{F}=\left\{F_{\gamma}: \gamma<\alpha\right\}$ for $\alpha<\omega_{1}$ and that the remaining conditions stated in (i) are fulfilled. Let $S$ denote the set of all functions $f: X \rightarrow \mathbb{R}$ such that $f \mid F_{\gamma}$ has the Baire property for each $\gamma<\alpha$. Then $C_{I} \subset S$ and $S$ is closed with respect to pointwise limits. Hence $B_{\gamma}(I) \subset S$ for each $\gamma<\omega_{1}$. Thus it suffices to show that $S \subset B_{1}(I)$. Let $f \in S$. By virtue of Kuratowski's result [K1] and Proposition 4, there exist functions $g_{\gamma}: F_{\gamma} \rightarrow \mathbb{R}$, $\gamma<\alpha$, of the Baire class 1, such that the set $\left\{x \in F_{\gamma}:\left(f \mid F_{\gamma}\right)(x) \neq g_{\gamma}(x)\right\}$ is of the first category in $F_{\gamma}$. Define $g: X \rightarrow \mathbb{R}$ by $g(x)=g_{\gamma}(x)$ for $x \in$ $F_{\gamma} \backslash F_{\gamma+1}, \gamma<\alpha$, and

$$
g(x)=0 \text { for } x \in\left(X \backslash F_{0}\right) \cup \bigcup_{\lambda<\alpha, \lambda \text { limit }}\left(\bigcap_{\gamma<\lambda} F_{\gamma} \backslash F_{\lambda}\right) .
$$

Since

$$
\{x \in X: f(x) \neq g(x)\} \cap F_{\gamma} \subset\left\{x \in F_{\gamma} \backslash F_{\gamma+1}:\left(f \mid F_{\gamma}\right)(x) \neq g_{\gamma}(x)\right\} \cup F_{\gamma+1},
$$

therefore $\{x \in X: f(x) \neq g(x)\} \cap F_{\gamma}$ is of the first category in $F_{\gamma}$. Thus

$$
\{x \in X: f(x) \neq g(x)\} \in M G R(\mathscr{F})=I \text {. }
$$

The function $g$ is of the Baire class 1 since for each open subset $U \subset \mathbb{R}$ we have $g^{-1}[U] \in \Sigma_{2}^{0}$. Indeed,

$$
g^{-1}[U]=\bigcup_{\gamma<\alpha}\left(g_{\gamma}^{-1}[U] \backslash F_{\gamma+1}\right) \quad \text { if } 0 \notin U,
$$

and

$$
\left(X \backslash F_{0}\right) \cup \bigcup_{\lambda<\alpha, \lambda \text { limit }}\left(\bigcap_{\gamma<\lambda} F_{\gamma} \backslash F_{\lambda}\right)
$$


must be added to the right side of the last equality, if $0 \in U$. Consequently, $f \in B_{1}(I)$ by Proposition 4 .

Case (ii). Use Propositions 2, 3, and 5.

Remark. At this moment we do not know which values between 1 and $\omega_{1}$ can be achieved by $r(I)$ when $I$ is not uniform. That question for non-uniform principal $\sigma$-ideals $I_{A}=\{E \subset X: E \subset X \backslash A\}$ where $A \subset X$ is uncountable was considered in [M3]. Note that $r\left(I_{A}\right)=\omega_{1}$ if $A$ contains a perfect set (see [M3, Theorem 6]). So, it would be interesting to examine the case when $A$ is uncountable and does not contain perfect sets.

\section{REFERENCES}

[B1] M. Balcerzak, A generalization of the theorem of Mauldin, Comment. Math. Univ. Carolin. 26 (1985), 209-220.

[B2] Classfication of $\sigma$-ideals, Math. Slovaca 37 (1987), 63-70.

[B3] _ Can ideals without ccc be interesting?, Topology Appl. 55 (1994), 251-260.

[KS] A. S. Kechris and S. Solecki, Approximation of analytic by Borel sets and definable countable chain conditions, Israel J. Math. (to appear).

[K] K. Kuratowski, Topology I, Academic Press, New York, 1966.

[K1] K. Kuratowski, Sur les fonctions représentables analytiquement et les ensembles de première catégorie, Fund. Math. 5 (1924), 75-86.

[L] H. Lebesgue, Sur les fonctions représentable analytiquement, J. Math. (6) 1 (1905).

[M1] R. D. Mauldin, $\sigma$-ideals and related Baire systems, Fund. Math. 71 (1971), 171-177.

[M2] - The Baire order of the functions continuous almost everywhere, Proc. Amer. Math. Soc. 41 (1973), 535-540.

[M3] _ The Baire order of the functions continuous almost everywhere II, Proc. Amer. Math. Soc. 51 (1975), 371-377.

[Mo] Y. N. Moschovakis, Descriptive set theory, North-Holland, Amsterdam, 1984.

Technical University of Lodz, Institute of Mathemathics, Al. Politechniki 11, 90-924 Lodz, Poland

E-mail address, M. Balcerzak: MBALCE@PLUNL051. bitnet

E-mail address D. Rogowska: DOROTARO@LODZ1.P.LODZ.PL 